\title{
LA ADOPCIÓN INTERNACIONAL, ESTUDIOS REFERENTES A LOS VÍNCULOS DE LOS ADOLESCENTES, BÚSQUEDA DE IDENTIDAD Y PERCEPCIÓN DEL APOYO SOCIAL PERCIBIDO CON LOS PARES
}

\section{INTERNATIONAL ADOPTION: STUDIES ON ADOLESCENT'S BONDS, SEARCH FOR IDENTITY AND SOCIAL SUPPORT RECEIVED FROM THE PEERS}

\author{
María José Martín Rodríguez \\ Trabajadora Social Autónoma, Miembro del LIPAI (Listado Intervención Profesional \\ en Adopción Internacional)
}

\begin{abstract}
Resumen: La adopción internacional es un fenómeno social que se ha dado en las últimas dos décadas en España, siendo adoptados más de sesenta mil niños de diferentes países. Muchos de ellos están o han estado en estos últimos años transitando el periodo evolutivo de la adolescencia. Se considera de importancia estudiar la adaptación de los adolescentes adoptados internacionalmente en nuestras familias, por las diferencias interculturales e interraciales que tienen con su entorno y las dificultades que tienen que abordar por su identidad de adoptado en la adolescencia, cobrando importancia las relaciones y apoyos sociales que tienen positivos en su entorno. Este artículo expone las investigaciones más relevantes y las variables que se han estudiado, con el objeto de tener una aproximación a las dificultades de los adolescentes y reflexionar sobre los apoyos sociales más positivos que reciben de sus iguales. Esta exposición está desarrollada en el marco de una tesis doctoral en curso en la Universidad de Deusto con el título "Los apoyos sociales entre iguales, de los adolescentes adoptados internacionalmente", desde la perspectiva del adolescente/joven.
\end{abstract}

Palabras Clave: Adolescente, Adopción Internacional, Apoyo social, Vínculo, Pares.

\begin{abstract}
The international adoption is a social phenomenon which has had a significant importance during the last two decades in Spain, where more than 60000 children have been adopted from different countries. Many of them are or have been passing through the evolutionary stage of adolescence. The adaptation of internationally adopted adolescents in our families is considered to be of great importance, because of the intercultural and interracial differences they have with their environment, and the difficulties they have to deal with, which can lead them to any kind of identity problems as adopted in adolescence. Consequently, social relationships and support in their environment have a positive impact on them. This article outlines the most relevant research and the variables that have been studied, in order to have an approach to the difficulties of adolescents and reflect about the most positive social supports they receive from their peers. This exposition is carried out within the framework of a doctoral thesis underway at the University of Deusto with the title "Social support among equals of internationally adopted adolescents", from the perspective of the adolescent / young person.
\end{abstract}

Keywords: Adolescent, International Adoption, Social support, Link, Peers.

| Recibido: 22/05/2018 | Revisado: 30/07/2018 | Aceptado: 21/01/2019 | Publicado: 30/01/2019

Correspondencia: María José Martín Rodríguez. Trabajadora social autónoma. Miembro del LIPAI.

Email: medeas@interbook.net.

Referencia normalizada: Martín-Rodríguez, M.J. (2018). La adopción internacional, estudios referentes a los vínculos de los adolescentes, búsqueda de identidad y percepción del apoyo social percibido con los pares. Trabajo Social Hoy, 86, 37-52. doi: 10.12960/TSH.2019.0003 


\section{INTRODUCCIÓN}

En las teorías del desarrollo todos los especialistas hacen mención a la crisis de la adolescencia, momento del desarrollo evolutivo en el que se enfrenta a numerosos cambios, no solo de índole físico, sino psíquico y en sus relaciones en el ámbito social. Erikson (1968) afirma que en la búsqueda de su identidad necesita, "un sentirse vivo y activo, ser uno mismo, la tensión activa y confiada y vigorizante de sostener lo que me es propio; es una afirmación que manifiesta una unidad de identidad personal y cultural", esta afirmación nos traslada la importancia de los aspectos culturales en la identidad del individuo, como resultado de lo biológico, lo psicológico y lo social. La identidad se forma entre el individuo y la sociedad y cómo este la enfrenta con las dificultades que va teniendo; este autor se distancia de las teorías freudianas del inconsciente poniendo el valor en el yo, que se forma en interrelación con los otros.

Es por ello, que las relaciones afectivas entre iguales o de pareja en los adolescentes y jóvenes son importantes en el desarrollo psicosocial, con directas implicaciones para la salud, bienestar y ajuste psicológico (Booth y Crouter 2006; Collins, 2003; Furman y Shaffer, 2003) (y son una fuente de apoyo social emocional e instrumental al estar presente en los momentos de ocio (Furman, Low y Ho, 2009) y de gran importancia en la construcción de la identidad (Collins, Welsh, y Furman, 2009; Furman y Shaffer, 2003).

Para el análisis de las relaciones humanas, se ha de destacar la teoría del apego. En la primera infancia el niño forma su psique en relación con la madre o su cuidador principal. Bowlby (1986) desarrolla la teoría del apego, analizando las relaciones de la primera infancia, estableciendo las bases del comportamiento infantil. Esta teoría analiza la calidad de la relación madre/hijo en los primeros años de vida y su influencia en la manera de relacionarse en etapas posteriores. Ainsworth (1962) confirma esta teoría empíricamente, con el experimento que se conoce como «la situación extraña», definiendo tres tipos de apego: el seguro, el evitativo y el resistente ante la ausencia de la figura de apego. Con esta misma teoría (Main y Salomon 1986) habla de un cuarto estilo: el desorganizado, que correlaciona con situaciones de maltrato y abandono (Fonagy, 2001; Oliva, 2004).

Aunque esta teoría del apego informa de los primeros momentos de la vida del niño y el mundo de la relación con los padres, como determinantes en la manera de relacionarse posteriormente; otros autores afirman que la resiliencia o la capacidad de superar las situaciones adversas, el buen trato, el apoyo social recibido y nuevas relaciones, moldean y modifican el apego, reparando y construyendo vínculos más seguros (Barudy, 2009; Brodzinsky, 2011, Cyrulnik, 2007).

Las investigaciones sobre apego en la primera infancia son numerosas, pero en el estudio del apego en adolescentes su número se reduce significativamente. De las más representativas tenemos las investigaciones que relacionan el estilo de apego 
con la autoestima (Holmes, 2011) y la adaptación social (Pinheiro y Mena, 2014); otros trabajos centran sus hallazgos en relación con las psicopatologías (Allen et al., 2007; Bowlby, 1986).

En la misma línea (Hazan y Shaver, 1987) estudian el apego en la relaciones de pareja adultas y otros autores posteriormente, las características de estas relaciones de apego y sus efectos en las relaciones de pareja tanto de adolescentes como de jóvenes y adultos (Brumbaugh, Fraley, Heffernan y Vicary, 2012; Mikulincer et al., 2002; Feeney, 1999; Fraley, 2002; Holland et al., 2012; Mikulincer y Shaver, 2003; Mikulincer y Shaver, 2007), habiendo una correspondencia positiva sobre el apego infantil y la manera de relacionarse en etapas posteriores.

\section{ADOPCIÓN Y APEGO}

Los niños adoptados han vivido una realidad distinta, han vivido un periodo más o menos largo en una institución, y han tenido distintos cuidadores y no han tenido una figura de apego estable. Esta adversidad marca la relación con sus padres adoptivos, que con diferentes herramientas y capacidades se enfrentan al reto de conseguir reparar su dolor y crear un vínculo seguro. Es por ello que las investigaciones buscan conocer las diferencias de vinculación/apego, por ser una pieza clave en su desarrollo.

En el ámbito de las investigaciones de apego sobre niños adoptados se ha de destacar las relacionadas con las dificultades que presentan a su llegada y la recuperación tras la adversidad. La mayoría de las investigaciones concluyen que, como norma general, la mayor exposición a la adversidad (niños que han estado más tiempo institucionalizados o han vivido situaciones traumáticas) tiene efectos más negativos en el desarrollo y más dificultades para recuperarse, (Beckett, Castle, Colvert, Kreppner, Mehta, Rutter, SonugaBarke y Stevens 2007; Fox, Guthrie, Marshall, Nelson, Smyke y Zeanah, 2007).

Sin embargo, en el ámbito de los adolescentes adoptados son muy pocas las investigaciones que tenemos y sobre todo de las adopciones trasnacionales. Es a partir del año 2000 cuando se inicia más investigación desde distintas disciplinas, con el objetivo de conocer los procesos de los adolescentes adoptados y los factores que entran en juego, de una manera explicativa y no descriptiva. Son áreas de estudio, lo biológico analizando la importancia de lo genético, en el ámbito de las neurociencias y se ha de destacar el estudio de las relaciones/vínculos y sus dificultades en la construcción de su identidad.

Una de sus dificultades consiste en las falsas creencias (González-Cuenca, Esteban y Molina, 2005). Con una muestra de 54 adolescentes adoptados con edades entre 11 a 18 años. Informan que tienen un peor rendimiento en la comprensión, entienden de manera literal, por lo que entienden mal los conceptos. 
Elovainio, Lapinleimu, Mäkipää, Matomäki, Raaska y Sinkkonen (2012), estudian la prevalencia en Finlandia de la asociación positiva entre victimización/intimidación y trastorno de apego reactivo, problemas de aprendizaje y dificultades en el lenguaje, de los adolescentes adoptados, concluyendo en la necesidad de intervenir para mejorar el apego tempranamente, en los niños que sufren trastorno de apego reactivo.

Abrines y colaboradores (2014), con una muestra de 168 niños adoptados internacionalmente, concluyen que el estilo de apego inseguro, fue ligeramente superior al seguro; observando que los niños que provenían de oriente de Europa tenían mayores tasas de apego inseguro; esto identifica un grupo de riesgo para posteriores intervenciones. Recientemente Grant-Marsney, Grotevant y Sayer (2015) en un estudio longitudinal de adoptados desde bebés, concluyen que el mayor apego a los padres predice un menor estilo evitativo durante la adolescencia, y la mayor cercanía de los padres a lo largo del tiempo se relaciona con menos ansiedad en las relaciones cercanas. Farr, GrantMarsney y Grotevant (2014), en un estudio de 167 jóvenes, analizan la importancia de la percepción de la apertura sobre la comunicación positiva sobre la adopción que se correlaciona positivamente con un apego seguro a sus padres adoptivos, siendo estas variables predictores de la significación positiva de los padres adoptivos y la satisfacción de las relaciones con los padres biológicos, estos dos estudios están realizados en EE.UU. dónde la apertura de la adopción y los contactos con la familia biológica, tiene largo recorrido.

\section{IDENTIDAD DEL ADOLESCENTE ADOPTADO TRASNACIONAL Y TRANSRACIAL. IMPORTANCIA DEL APOYO SOCIAL}

Para el abordaje de este tema tan complejo, específicamente el de las adopciones trasnacionales y transraciales en la etapa de la adolescencia, se ha de tener en cuenta que el adolescente adoptado trasnacional en su búsqueda de identidad tiene la tarea de confrontar su identidad de adoptado, su origen y las diferencias étnicas, con relación a las dos culturas, la adoptiva y la de origen Baden, Grotevant, Lee, Mohanty, Pinderhughes, y Rosnati, (2015). Debe encontrar un equilibrio entre sus múltiples identidades. Por esa circunstancia, el apoyo social a lo largo de la vida, formal e informal, puede ser una pieza clave para ayudar al adolescente Cava y Musitu (2003) concluyen en su estudio que el apoyo social desempeña un papel muy importante durante la adolescencia, por tanto la falta de este apoyo puede hacernos entender las numerosas dificultades que tienen. El vínculo/apego seguro del adoptado con su familia biológica y posteriormente con la familia adoptiva es un apoyo y tiene implicaciones a lo largo de su vida, pero en la adolescencia en la búsqueda de su autonomía y en las relaciones interpersonales, es un momento crítico en la creación de vínculos con sus pares. Las personas adoptadas, por el desconocimiento de partes de su historia antes de la 
adopción, tienen la dificultad de construir una narrativa coherente que vincule su historia pasada, en el presente, para proyectar su futuro y esto le hace tener una desventaja con sus pares.

Es por ello que en los últimos años se ha buscado respuestas a diversos interrogantes, entre otros, cómo influye el conocimiento por parte de los adoptados, de sus antecedentes genéticos. En este campo las investigaciones actuales, se centran en los factores biológicos y la influencia en el desarrollo de los adoptados internacionalmente; Grotevant y May (2018) concluyen que el conocimiento de los antecedentes familiares, y por tanto todo aquello que pueda afectar en el futuro del adoptado en el campo de la salud, tiene un papel relevante en los objetivos y proyectos, así como en poner los medios para conseguirlos. El conocimiento de la información genética por parte de los adoptados y por tanto de su salud influye en la formulación de proyectos en su vida. La información de la salud tiene una relación positiva en la búsqueda personal de medios para conseguir los proyectos, independientemente de su estado de salud actual. En esta línea Dunbar, Fiorenzo, Grotevant y Lo (2017) estudian las características comunes de la salud de los niños adoptados, para ayudar a los adoptados en ausencia de información de los padres biológicos.

Es de gran importancia un estudio finlandés sobre antecedentes genéticos de esquizofrenia en niños adoptados. Concluyen los autores que aunque la genética es un factor importante para desarrollar una enfermedad, es la interacción de genética y ambiental, la que determina el desarrollo de esta (Lahti, Läksy, Moring, Sorri, Tienari, Wahlberg y Wynne, 2004), de esta manera las dinámicas familiares son un factor muy importante en la enfermedad mental, esto ayuda para no atribuir directamente una psicopatología a una carga genética, sino en interacción con su entorno, dinámicas familiares y relaciones interpersonales.

\section{IDENTIDAD ÉTNICA Y BÚSQUEDA DE ORÍGENES}

Muchas de las familias que han adoptado no han podido prever el efecto de las diferencias culturales y étnicas en su entorno, por tener poca experiencia los/as profesionales y no haber sido apoyados adecuadamente. En ocasiones la elección de muchas familias que han optado por este tipo de adopción ha sido motivada por la viabilidad de realizar el proyecto de adopción en un tiempo reducido y con un ofrecimiento de un niño pequeño. La percepción de las familias ha sido poco real, algunos autores (Dwyer, 2013) hablan de "padres miopes". Los padres tenían la percepción de una sociedad más avanzada e inclusiva. Los avances en los medios de comunicación, les ayudaba a los padres a tener un mayor acercamiento al origen de sus hijos/as y comentaban que en los colegios no se verían diferentes porque había muchos niños de otras etnias por la inmigración. No obstante, las investigaciones constatan que existen dificultades que tienen relación con su etnicidad y cultura, en la formación de la identidad. 
Para una mayor complejidad de esta cuestión, recientemente la ley Española 26/2015 en materia de adopción, entre otras medidas, regula con más detalle la capacidad de los adoptantes y se incorpora una definición de la idoneidad para adoptar; creando la figura la adopción abierta, que permite al adoptado mantener relación con su familia de origen a través de visitas o comunicaciones, lo que ha de ser acordado por el juez y se refuerza el derecho de acceso a los orígenes de las personas adoptadas. Es un cambio cualitativo en la adopción y esto hace pertinente conocer lo que se ha investigado y hacer propuestas políticas adaptadas para apoyar a los adolescentes adoptados y sus familias. Esta cuestión ha modificado la percepción sobre la adopción por parte de los padres.

Es de actualidad una de las primeras investigaciones que se han realizado en adopciones internacionales, porque nos acerca a la realidad de las necesidades de los niños y las dificultades en las familias; este estudio concluye que las familias se sienten insatisfechas, cuando los hijos adoptados empiezan a distanciarse emocionalmente y buscan el acercamiento a sus iguales, momento en que por las diferencias étnicas y la condición de adoptado, aparecen dificultades. Este estudio se realizó con una amplia muestra de niños/as vietnamitas adoptados en EE.UU. (Kirk, 1964).

En las recientes líneas de investigación se ha de destacar el meta análisis de Aaro, Askeland, Hysing, La Greca, Sivertsen y Tell (2017). En este estudio se incluyeron once estudios europeos, con un total de 17919 adoptados y 1090289 pares no adoptados, concluyendo que aunque la mayoría de los adolescentes adoptados internacionalmente tienen un buen ajuste social, hay una mayor prevalencia en salud mental en comparación a sus pares no adoptados.

Grotevant y colaboradores (2015), en una revisión de seis artículos destacan la importancia de la socialización étnico-racial y la identidad étnica entre los adoptados transraciales e internacionales. Aportando en sus conclusiones que la familia, los compañeros, la comunidad y la cultura de acogida son contextos de socialización importantes y es en los procesos de relación con los otros donde se promueve la identidad étnica. Estos autores destacan la importancia de los padres que dan un apoyo apropiado para el desarrollo de la identidad étnica positiva.

Latorre (2013) nos refiere sobre la adopción:

"Este proceso requiere un sobre-esfuerzo por parte de los adoptados y por parte de los adoptantes. Las familias adoptantes con diferentes actitudes y comportamientos pueden mejorar el apego inseguro de quienes han adoptado y ayudar de esta forma a reparar el dolor de su hijo o hija, generando un apego seguro que permita desarrollar una identidad positiva". 
A pesar de los pocos conocimientos sobre cómo se perciben los diferentes grupos transraciales adoptados en las culturas dominantes, se reconoce que las percepciones sociales negativas hacia su cultura de nacimiento impactan en el desarrollo de la identidad (Garber et al., 2015).

En esta línea (Garber y Grotevant 2015) estudiaron la percepción que tenían 153 personas adultas sobre microagresiones por su condición de adoptados, definiendo estas tipologías: silencio, preguntas excesivamente intrusivas, asunción de bionormalidad, confusión/ignorancia recurrente, divisiones internas, excluirlos del grupo, uso de su adopción, falsas creencias, no reconocimiento de su identidad, hacerse portavoz de la adopción, pensar en que el ser adoptado es raro, estereotipos negativos sobre sus padres biológicos, adoptado por ser huérfano, representación social negativa de la adopción. Es indudable que la falta de instrumentos y habilidades para responder a estos estereotipos generan dificultades y muchas de ellas pueden ser adquiridas en la infancia con los padres adoptivos, pero en la adolescencia requerirá de un discurso en el que pueda incluir su historia pasada de una manera positiva con sus iguales.

Se empieza a constatar que los adolescentes jóvenes adoptados en España, tienen la necesidad de conocer su historia y en algunas ocasiones tener relación con sus padres biológicos, así como los adoptados trasnacionalmente realizan preguntas y solicitan un acercamiento a sus orígenes y en ocasiones desean conocer a sus familias biológicas. Brodzysky (2011), nos dice que los adoptados, piensa en sus orígenes aunque no lo manifiesten.

Dentro de las investigaciones que tienen relación con la confrontación de la identidad adoptiva. Dunbar y colaboradores (2017) en un estudio reciente longitudinal, sobre la identidad y el ajuste, informan que los adolescentes adoptados con una identidad inestable tienen niveles significativamente más altos de problemas internalizantes: ansiedad y depresión en la juventud y su adultez.

Grotevant, Martin McDermott (2014) estudian los procesos de familias adoptivas que tienen contacto con la familia biológica, las familias adoptivas homosexuales y lesbianas y la socialización racial en las familias adoptivas transraciales concluyendo que todo afecta al desarrollo social de los niños adoptados hasta la edad adulta.

Anteriormente había sido estudiado en EE.UU. por (Grotevant, Skinner-Drawz, Von Korff, Wrobel, 2011), la apertura comunicativa de las madres adoptivas y esta variable se asoció positivamente con el grado de búsqueda de información en la juventud de los adoptados. El grado de búsqueda de información entre la adolescencia y la juventud aumentó para la mayoría de los adoptados, se encontraron diferencias en cuanto al género, las mujeres buscaban menos. Se observó que el proceso de búsqueda era dinámico, en distintas etapas de la adolescencia y juventud, cambiaba su interés. 
En la misma línea Grotevant y colaboradores (2013) investigaron la motivación en la búsqueda de orígenes, de niños adoptados de la misma raza que los padres. Concluyendo que el nivel de motivación que tenían en la búsqueda de orígenes estaba relacionado positivamente con la búsqueda de información y la percepción que tenía el adolescente sobre las dificultades que encontraba.

Ayers-Lopez, Grotevant, Mcroy, Wrobel (2013) en un estudio longitudinal sobre la apertura de comunicación de la adopción, concluyen que los adolescentes y jóvenes adoptados que tienen contacto con su familia biológica están más satisfechos y tienen un mejor ajuste y desarrollo de su identidad que los que no tenían contacto. En este mismo estudio se observó que las madres biológicas que tenían contacto, tenían elaborado mejor el duelo y estaban más satisfechas, independientemente del nivel de contacto. Se consideró que los parientes, tanto adoptivos como biológicos, necesitaban de un aprendizaje en habilidades sociales, para un buen compromiso y tener flexibilidad.

\section{APOYO SOCIAL ENTRE IGUALES DE LOS ADOLESCENTES ADOPTADOS}

El apoyo social es un pilar del bienestar individual, los seres queridos ayudan ante las dificultades, el saber que se puede contar con las personas significativas tranquiliza, por eso es una necesidad del ser humano tener relaciones para sentirse querido y aceptado. Cava y Musitu (2003) estudiaron la percepción del apoyo social de los adolescentes, concluyendo que, en general, tiene una gran importancia la percepción de apoyo de los padres durante la adolescencia. «El apoyo social, ciertamente, desempeña un rol fundamental durante esta transición». Sin embargo, la red social del adolescente cobra mucha importancia sobre todo la del mejor amigo y de la pareja. Cabe esperar que en los adolescentes adoptados pase también, no obstante parten de diferencias y dificultades distintas por su condición de adoptado y pueden requerir de un mayor apoyo.

De ahí la importancia de todos los estudios que nos aporten conocimientos en esta área. Grotevant y Lo (2017), en una revisión de investigaciones con tres enfoques: la apertura en el contacto con las familias biológicas, la socialización racial, cultural en la relaciones transraciales y la experiencia en la crianza de padres adoptivos gays y lesbianas, concluyen que, en general, el apoyo recibido ante los problemas que les surgen en las relaciones predice un mejor ajuste infantil de los adoptados que las diferencias por apertura ante la adopción o diferencia de padres adoptivos gays, lesbianas.

Bakermans-Kranenburg, Beijersbergen, Juffer y van IJzendoorn (2012), en un estudio longitudinal de 125 niños adoptados a una edad temprana hasta los catorce años, investigaron el apoyo sensible de los padres y se observó que las madres de los adolescentes que tenían un apego seguro, tenían más apoyo sensible en los momentos 
de discusión y conflicto que los adolescentes inseguros, y que el apoyo sensible en la infancia predijo un apego seguro. No obstante, las madres que habían dado un apoyo menos sensible en la infancia pero habían dado un mejor apoyo a sus hijos adolescentes, predijo un cambio de apego inseguro a un apego seguro. Por lo que el estudio concluye que el apoyo tanto el temprano como en la adolescencia son importantes en los primeros catorce años de vida.

En el ámbito de las relaciones con otros adoptados tenemos este estudio de investigación-acción con interés profesional para un diseño de intervención. Garber y colaboradores (2015) en un estudio realizado con un grupo de adolescentes adoptados, dónde todos tienen asignado un mentor adoptado universitario de su misma etnia; explorando la identidad étnica individual, la comunicación sobre su adopción/etnicidad, las relaciones sociales fuera de la familia, concluyen que los mentores percibían que el menor que se les había asignado tenía una identidad ambivalente étnica, pero afirmaban que fue muy positiva la experiencia.

Farr, Flood y Grotevant (2016), analizan tres estudios sobre el papel de los hermanos de los adoptados vistos desde la adolescencia a la adultez en una continuación del estudio de Berge y colaboradores (2006), sobre adolescentes adoptados y sus hermanos adoptivos con contacto con la familia biológica desde su nacimiento; en el primer estudio encontraron la evidencia de cambios en la manera de establecer el contacto. En el segundo estudio, los adoptados con hermanos (adoptados o no) que estaban más involucrados, en contacto con la familia biológica de los adoptados, los resultados muestran que la conducta de los adoptados fue más favorable que en los adoptados cuyos hermanos no se habían implicado en el contacto. Y en el tercer estudio, niños adoptados con hermanos que también fueron adoptados, los resultados mostraron que los niños adoptados percibían más positivamente su adopción cuando los hermanos expresaban sentimientos positivos similares sobre su adopción. Concluyen: que las experiencias de la adopción de los hermanos son importantes para la experiencia de adopción de los demás. En el estudio se observa que es un factor mediador y de apoyo el que los hermanos se involucren en las relaciones con su familia de origen y las experiencias sobre su adopción sean positivas.

Y para concluir se resalta la investigación sobre la percepción de las familias adoptivas y apoyos recibidos, ante las dificultades educativas; Elovainio, Lapinleimu, Mäkipää, Matomäki, Raaska y Sinkkonen (2012) concluyen que hay un mayor estrés en algunas familias homosexuales, con hijos/as adolescentes adoptados, por el estigma social asociado a la orientación sexual, con repercusión en el proceso adoptivo. El estigma social por la diferencia de orientación sexual es un factor que hay que valorar en algunos contextos sociales y tenerlo presente en la investigación y en la intervención. 


\section{CONCLUSIONES}

La noción de identidad se refiere a un espacio intermedio entre los procesos individuales y sociales: "de un lado, un sentimiento de individualidad -ser una persona única- y del otro, un sentimiento de vinculación ligado al contexto social que el propio sujeto reconoce y asume» (Erikson, 1968), por tanto, los estudios e investigaciones deben ser referidos a un espacio temporal de lo social. Contamos en estos momentos con escasas investigaciones que nos ayuden a comprender los procesos de los adolescentes adoptados transnacionalmente, en España, por haberse dado de una manera masiva y con numerosos cambios en el ámbito social.

De las investigaciones expuestas se constata la necesidad de intervenir y apoyar a las familias y a los adolescentes adoptados, en distintos planos, desde lo macro social impulsando políticas que ayuden a poder mejorar el acercamiento a sus orígenes e impulsando políticas de atención a la diversidad.

Es necesaria la intervención directamente en las instituciones, dando formación a los profesionales e incorporando prácticas que no patologicen o estigmaticen a los niños adoptados. Conocer cómo son las relaciones entre iguales de los adolescentes adoptados, con quién hablan de su adopción y qué es lo que hablan de ello, nos aporta información para ayudarles en la construcción de su historia y generar un discurso adaptado a su realidad que les permita tener respuestas ajustadas y adquirir habilidades.

Es necesario conocer las dificultades de relación, en el entorno social y cultural en donde están. Porque puede ser para ellos violento y poco respetuoso con sus derechos. La falta de sensibilidad, las frases estereotipadas y desafortunadas les colocan en una situación de desventaja social, que hace necesaria una intervención. Las dificultades para curar las heridas de su historia pasada, les hacen tener a su vez dificultades escolares y es en este ámbito dónde surgen muchos de los conflictos, por no saber atender a esta diversidad de alumnado.

De Rancourt y colaboradores (2012) en un estudio de 21 familias, en Francia, puso en relevancia que las familias tenían dificultades en el ámbito sanitario porque los profesionales de estos servicios frecuentemente estigmatizan a estas familias y no estaban atentos a los procesos familiares, concluyendo que es un buen apoyo la escucha de sus dificultades con profesionales preparados en este ámbito, proponiendo cambios de políticas socio-sanitarias. Es por ello que la sensibilidad/formación en el ámbito sanitario es fundamental

A nivel micro social, la familia cobra su mayor protagonismo siendo para la práctica clínica los que facilitan que los niños puedan relacionarse con normalidad para poder conseguir redes de apoyo (Cyrulnik, 2001; Barudy y Dantagnam, 2005), por ello el apoyo a la familia es una pieza clave para la integración de los menores. 
Barudy y Dantagnam (2005) y Guédeney (1998) nos hablan del apoyo social, afectivo con adultos y pares, que junto con los padres y la familia extensa, son una fuente importante para desarrollar un buen apego, obtener valores, participar en actividades sociales positivas, creativas y con humor todo ello ayuda a una buena integración. En la misma línea Cyrulnik $(2001,2003)$ nos dice que los adultos significativos constituyen verdaderos factores de integración del niño por la calidad de apoyo afectivo instrumental que aportan al generar conversaciones que les permite encontrar nuevos significados a las circunstancias que provocaron sus sufrimientos. La resiliencia secundaria se entiende como la capacidad de una persona de sobreponerse los desafíos y adversidades de la vida es gracias al apoyo afectivo social (Barudy y Dantagnam, 2010).

Se deben realizar políticas que ayuden a un mayor apoyo a las familias y a los adolescentes adoptados, analizar sus necesidades e intervenir en el entorno con proyectos interculturales por su vulnerabilidad, según demuestran las investigaciones que se han referido. Así como realizar políticas que hagan posible el acercamiento a las familias de origen de los niños.

Los estudios futuros deben evaluar la efectividad de las intervenciones dirigidas al desarrollo de la identidad étnica y adoptiva de los/as adoptados/as y por tanto será también importante el estudio de las relaciones con los iguales que son también adoptados/as y si son una fuente de apoyo emocional.

\section{BIBLIOGRAFÍA}

Aaro, L.E., Askeland, K.G., Hysing, M., La Greca, A.M., Sivertsen, B., \& Tell, G. S. (2017). Mental health in internationally adopted adolescents: a meta-analysis. Journal of the American Academy of Child \& Adolescent Psychiatry, 56(3), 203-213. doi:10.1016/j.jaac.2016.12.009

Allen, J.P., Porter, M., McFarland, C., McElhaney, KB, y Marsh, P. (2007). La relación de la seguridad del apego con las relaciones paternales y de pares de los adolescentes, la depresión y el comportamiento externo. Desarrollo del niño, 78(4), 1222-1239.

Abrines, N., Barcons, N., Brun, C., Fumadó, V., Marre, D., \& Sartini, C., (2014). Attachment and adaptive skills in children of international adoption. Child \& family social work, 19(1), 89-98.

Ainsworth, M. (1962). Los efectos de la privación materna: una revisión de los hallazgos y la controversia en el contexto de la estrategia de investigación. En M. Ainsworth, R. Andry, R. Harlow, S. Lebovici, M. Mead y G. Prugh, Privación de cuidado materno: una reevaluación de sus efectos (pp. 97-159). Ginebra, Suiza: Organización Mundial de la Salud.

Ayers-López, S., Grotevant, H.D., McRoy, R.G., y Wrobel, G.M. (2013). Contact bet- 
ween adoptive and birth families: Perspectives form tne Minnesota/Texas Adoption Research Project. Child development perspectives, 7(3), 193-198.

Baden, A.L., Grotevant, H.D., Lee, R.M., Mohanty, J., H., Pinderhughes, E.E., \& Rosnati, R. (2015). New Trends and Directions in Ethnic Identity Among Internationally Transracially Adopted Persons: Summary of Special Issue. New directions for child and adolescent development, 150, 91-95.

Bakermans-Kranenburg, M.J., Beijersbergen, M.D., Juffer, F., \& van IJzendoorn, M. H. (2012). Remaining or becoming secure: parental sensitive support predicts attachment continuity from infancy to adolescence in a longitudinal adoption study. Developmental psychology, 48(5), 1277. doi: 10.1037/a0027442

Barudy, J. (2009). Conferencia presentació a les Jornadas Europeas sobre Resiliencia. Institut Francès de Barcelona, 19 i 20 de març.

Barudy, J., y Dantagnana, M. (2005). Los buenos tratos a la infancia. Parentalidad, apego y resilencia. Barcelona: Gedisa.

Barudy, J., y Dantagnan, M. (2010). Los desafíos invisibles de ser padre o madre: Manual de evaluación de las competencias y la resiliencia parental. Barcelona: Editorial Gedisa.

Booth, A., y Crouter, A.C. (2006). Romance and Sex in Adolescence and Emerging Adulthood: Risk and Opportunities. Mahwah, N.J.: Erlbaum.

Bowlby, J. (1986). Vínculos afectivos: formación, desarrollo y pérdida. Madrid: Morata.

Brodzinsky, D.M. (2011). Children's understanding of adoption: developmental and clinical implications. Prof Psychol Res Pr, 42, 200-207.

Brodzinsky, D.M. (2011). Children's understanding of adoption: developmental and clinical implications. Prof Psychol Res Pr, 42, 200-207.

Brodzinsky, D., y Palacios, J., (2010). Adoption research: Trends, topics, outcomes. International Journal of Behavioral Development, 34(3), 270-284.

Beckett, C., Castle, J., Colvert, E., Kreppner, J., Mehta, M., Rutter, M., Stevens, S., y Sonuga-Barke, E.J. (2007). Effects of profound early institutional deprivation: An overview of findings from a U.K. longitudinal study of Romanian adoptees. European Journal of Developmental Psychology, 4, 332-350. doi: 10.1080/17405620701401846

Berge, J. M., Mendenhall, T. J., Wrobel, G. M., Grotevant, H.D., y McRoy, R.G. (2006). Adolescents' feelings about openness in adoption: Implications for adoption agencies. Child Welfare, 85(6), 1011.

Cava, M.J., y M. Musitu, G., (2003). El rol del apoyo social en el ajuste de los adolescentes. Psychosocial intervention, 12(2). 179-192.

Collins, W.A. (2003). More tan myyh: The developmental significante of romantic relationing during adolescence. Journal of Personality, 70, 963-1008.

Collins, W. A., Welsh, D.P., y Furman, W. (2009). Adolescent romantic relationships. Annual review of psychology, 60, 631-652.

Cuenca, A.M. G., Esteban, C.B., y Molina, M.F. (2005). La comprensión de creencias falsas y de sentidos no literales en adolescentes adoptados. Psicothema, 17(1), 43-48.

Cyrulnik, B., (2001). La maravilla del dolor: el sentido de la resiliencia. Barcelona: Ediciones Granica S.A. 
Cyrulnik, B., Aúz, T.F., y Eguibar, B. (2003). El murmullo de los fantasmas: volver a la vida después de un trauma. Barcelona: Gedisa editorial.

Cyrulnik, B. (2007). De cuerpo y alma. Neuronas y afectos: la conquista del bienestar. Barcelona: Gedisa.

De Rancourt, F., Labbe, A., Laurichesse, H., Lesens, O., Poirier, V., Schmidt, A., F., y Vorilhon, P. (2012). Health care support issues for internationally adopted children: A qualitative approach to the needs and expectations of families. PLoS One, 7(2) doi:http://dx.doi.org.proxy-oceano.deusto.es/10.1371/journal.pone.0031313

Dunbar, N.D., Fiorenzo, L., Grotevant, H.D. \& Lo, A.Y.H., (2017). Adoptive identity and adjustment from adolescence to emerging adulthood: A person-centered approach. Developmental psychology, 53(11), 2195-2204. doi: 10.1037/dev0000352

Dwyer, J.G. (2013). Inter-Country Adoption and the Special Rights Fallacy. U. Pa. J. Int'l L., 35, 189.

Elovainio, M., Lapinleimu, H., Mäkipää, S., Matomäki, J., Raaska, H., \& Sinkkonen, J. (2012). Internationally adopted children in Finland: parental evaluations of symptoms of reactive attachment disorder and learning difficulties-FINADO study. Child: care, health and development, 38(5), 697-705. doi: 10.1111/j.1365-2214.2011.01289.x

Erikson E. (1968). Identidad, Juventud y Crisis. Buenos Aires: Paidós.

Farr, R.H., Flood, M. E., \& Grotevant, H.D. (2016). The role of siblings in adoption outcomes and experiences from adolescence to emerging adulthood. Journal of Family Psychology, 30(3), 386. doi: 10.1037/fam0000173

Farr, R.H., Grant-Marsney, H.S., \& Grotevnt, H.D. (2014). Adoptees contact with birth parents birth parents in emerging adulthood: The role of adoption communication and attachment to adoptive parents. Family process, 53(4), 656-671. doi: 10.1111/famp.12069

Feeney, J.A. (1999). Adult attachment, emotional control, and marital satisfaction. Personal Relationships, 6(2), 169-185.

Fonagy, P. (2001). La teoria dell'attaccamento è proprio diversa da quella psicoanalitica? Punti di contatto e divergenze tra i due paradigmi. Fonagy P., Target M.: Attaccamento e funzione riflessiva. Raffaello Cortina, Milano, 3-26.

Fraley, R.C. (2002). Attachment stability from infancy to adulthood: Meta-analysis and dynamic modeling of developmental mechanisms. Personality and social psychology review, 6(2), 123-151.

Fraley, R.C. (2002). Attachment stability from infancy to adulthood: Meta-analysis and dynamic modeling of developmental mechanisms. Personality and social psychology review, 6(2), 123-151.

French, Q., Garber, K.J., \& Grotevant, H.D. (2015). Participation in the Adoption Mentoring Partnership: Mentors' Experiences of Ethnic Identity. New directions for child and adolescent development, 150, 77-89. doi: 10.1002/cad.20123

Furman, W., Low, S., \& Ho, M.J. (2009). Romantic experience and psychosocial adjustment in middle adolescence. Journal of Clinical Child \& Adolescent Psychology, 38(1), 75-90. doi: 10.1080/15374410802575347 
Furman, W., \& Shaffer,L. (2003). The role of romantic relationships in adolescent development.In P. Florsheim (Ed. ), Adolecent romantic relations and sexual beavior: Theory, research, and practical implications (pp. 3-22).

Garber, K.J., \& Grotevant, H.D. (2015). "YOU Were Adopted?!" Microaggressions Toward Adolescent Adopted Individuals in Same-Race Families. The Counseling Psychologist, 43(3), 435-462. doi: 10.1177/0011000014566471

Garber, K.J., \& Grotevant, H.D. (2015). "YOU Were Adopted?!" Microaggressions Toward Adolescent Adopted Individuals in Same-Race Families. The Counseling Psychologist, 43(3), 435-462. doi: 10.1177/0011000014566471

Grant- Marsney, H.A., Grotevanta, H.D., \& Sayer, A.G. (2015). Links between Adolescents Closeness to Adoptive Parents and Attachment Style in Young Adulthood. Family relations, 64(2), 221-232. doi: 10.1111/fare.1211

Grotevant, H.D., \& Lo, A.Y. (2017). Adoptive parenting. Current opinion in psychology, 15, 71-75. doi: 10.1016/j.copsyc.2017.02.020

Grotevant, H. \& May, T., (2018). Autonomy, well-being, and the value of genetic testing For adopted persons, In HEC Forum 30,283-295.

Grotevant, H.D. \& McDermott, J.M. (2014). Adoption: Biological and social processes linked to adaptation. Annual review of psychology, 65, 235-265. doi: 10.1146/ annurev-psych-010213-115020

Wrobel, G.M., Grotevant, H.D., Samek, D.R., \& Korff, L.V. (2013). Adoptees' curiosity and information-seeking about birth parents in emerging adulthood: context, motivation, and behavior. International journal of behavioural development, 37(5), 441-445. doi: 10.1177/0165025413486420

Grotevant, H.D., Skinner-Drawz, B.A., Von Korff, L., \& Wrobel, G. M., (2011). The role of adoption communicative openness in information seeking among adoptees from adolescence to emerging adulthood. Journal of Family Communication, 11(3), 181-197. doi: 10.1080/15267431003656587

Guédeney, A. (1998). Les enjeux d'une classification et ses liens à la psychopathologie chez le jeune enfant. Devenir, 10(1), 17-25.

Hazan, C., \& Shaver, P. (1987). Romantic love conceptualized as an attachment process. Journal of personality and social psychology, 52(3), 511.

Heffernan, M.E., Fraley, R.C., Vicary, A.M., \& Brumbaugh, C.C. (2012). Attachment features and functions in adult romantic relationships. Journal of Social and Personal Relationships, 29(5), 671-693. doi: 10.1177/0265407512443435

Holmes, J. (2011). Teoria del apego y psicoterapia. En busca de la base segura. Bilbao: Desclée de Brouwer.

Holland, A.S., Fraley, R.C., \& Roisman, G.I. (2012). Attachment styles in dating couples: Predicting relationship functioning over time. Personal Relationships, 19(2), 234246. doi:10.1111/j.1475-6811.2011.01350.x

Kirk, H. D. (1964). Shared fate: A theory of adoption and mental health. London: CollierMacmillan; New York: The Free Press of Glenco [c1964].

Lahti, I., Läksy, K., Moring, J., Sorri, A., Tienari, P., Wahlberg, K.E., \& Wynne, L. C., 
(2004). Genotype-environment interaction in schizophrenia-spectrum disorder: Long-term follow-up study of Finnish adoptees. The British Journal of Psychiatry, 184(3), 216-222.

Latorre, F.L. (2013). Trabajando la identidad positiva con las personas adoptadas. Familias, apegos y vínculos como estrategias de consolidación de la identidad. Cuadernos de psicomotricidad, (46), 7-20.

Main, M. \& Solomon, J. (1986). Discovery of an insecure-disorganized / disoriented attachment pattern. En T.B. Brazelton and M.W. Yogman (ed.), Affective Development in infancy, (pp 95-124). Norwood, N J: Ablex.

May, T., \& Grotevant, H. (2017, December). Autonomy, Well-Being, and the Value of Genetic Testing for Adopted Persons. In HEC Forum (pp. 1-13). Springer Netherlands. doi: 10.1007/s10730-017-9345-0

Mikulincer, M., Florian, V., Cowan, P.A., \& Cowan, C.P. (2002). Attachment security in couple relationships: A systemic model and its implications for family dynamics. Family process, 41(3), 405-434.

Mikulincer, M., \& Shaver, P.R. (2003). The attachment behavioral system in adulthood: Activation, psychodynamics, and interpersonal processes. Advances in experimental social psychology, 35, 56-152.

Mikulincer, M., \& Shaver, P.R. (2007). Attachment in adulthood: Structure, dynamics, and change. Guilford Press.

Ministerio de Justicia. Ley 26/2015, de 28 de julio, de modificación del sistema de protección a la infancia y a la adolescencia. BOE nº 180 del miércoles 29 de julio de 2015.

Nelson, C.A., Zeanah, C.H., Fox, N.A., Marshall, P.J., Smyke, A.T., \& Guthrie, D. (2007). Cognitive recovery in socially deprived young children: The Bucharest Early Intervention Project. Science, 318(5858), 1937-1940. doi: 10.1126/science.1143921

Oliva Delgado, A. (2004). Estado actual de la teoría del apego. Revista de Psiquiatría y Psicología del Niño y del Adolescente, 4(1), 65-75.

Pinheiro, M.C., y Mena, M.P. (2014). Padres, profesores y pares: contribuciones para la autoestima y coping en los adolescentes. Anales de Psicología, 30(2), 656. doi: 10.1016/j.rmta.2015.05.006 
